\title{
Frequency-independent radiation modes of interior sound radiation: Experimental study and global active control
}

\author{
C. Hesse ${ }^{\mathrm{a}, *}$, V. Papantoni ${ }^{\mathrm{a}}$, S.Algermissen ${ }^{\mathrm{b}}$, H.P. Monner ${ }^{\mathrm{b}}$ \\ ${ }^{a}$ Institute of Composite Structures and Adaptive Systems, German Aerospace Center \\ (DLR), Sportallee 54a, 22335 Hamburg, Germany \\ ${ }^{b}$ Institute of Composite Structures and Adaptive Systems, German Aerospace Center
}

(DLR), 38108 Braunschweig, Germany

\begin{abstract}
Active control of structural sound radiation is a promising technique to overcome the poor passive acoustic isolation performance of lightweight structures in the low-frequency region. Active structural acoustic control commonly aims at the suppression of the far-field radiated sound power. This paper is concerned with the active control of sound radiation into acoustic enclosures. Experimental results of a coupled rectangular plate-fluid system under stochastic excitation are presented. The amplitudes of the frequency-independent interior radiation modes are determined in real-time using a set of structural vibration sensors, for the purpose of estimating their contribution to the acoustic potential energy in the enclosure. This approach is validated by acoustic measurements inside the cavity. Utilizing a feedback control approach, a broadband reduction of the global acoustic response inside the enclosure is achieved.
\end{abstract}

Keywords: Active control, ASAC, Radiation modes, Fluid-structure interaction

\section{Introduction}

Most recent approaches for the active control of sound radiation of vibrating structures into enclosed spaces are based on structural mode sensing 1, 2]. This requires a priori knowledge of the structural mode shapes which can necessitate a high identification effort. In Hesse et al. [3, the structural vibration is decomposed into a set of interior radiation modes. The interior radiation modes are orthogonal functions that describe vibration modes of the structure, such that the contribution from each one of them to the acoustic potential energy in the enclosed fluid is uncoupled from any other. The interior radiation modes

\footnotetext{
* Corresponding author

Email address: christian.hesse@dlr.de (C. Hesse)
} 
calculated in [3] do not presume the knowledge of structural mode shapes and are independent of frequency, as they are a subset of the cavity modes at the structural interface. Since the radiation modes are orthogonal with respect to the acoustic potential energy, their reduction by active means will lead to a global reduction of the enclosed sound field.

The mechanisms of the active control of the acoustic potential energy are investigated in a series of publications by Snyder et al. [4, 5] using secondary control inputs in the fluid and on the structure. Using an analytical model with vibration control sources, e.g. a point force, the two mechanisms of modal suppression and modal rearrangement can occur. The mechanism of modal suppression consists in the reduction of structural modal amplitudes, which are efficiently coupled to the fluid modes. Modal rearrangement on the other hand, is based on the fact, that one fluid mode is in general coupled to more than one structural mode. If this is the case, the structural modes are rearranged in a way, that the coupling to the cavity mode is less efficient.

Many approaches, regarding experimental realizations of active control of structural sound radiation into enclosed spaces, deal with the reduction of local sound pressures [6, 7, , 8] or local energy density [9]. Kim and Brennan investigate the active control of harmonic and random sound transmission into an acoustic enclosure [10, 11]. 11 microphones inside the cavity are used as error sensors and the acoustic potential energy is estimated based on the sum of the squared pressure amplitudes. A structural actuator is shown to be more successful in controlling the structural vibration modes than an acoustic actuator.

The approach of local pressure control does not necessarily result in a global reduction of sound radiation. Although the active control with structural error sensing based on radiation modes is investigated numerically [1, 2, an experimental study using a set of radiation modes and yielding a broadband reduction of the acoustic potential energy is not known to the authors. A reason for the absence of experimental work may be found in the frequency-dependence of the resulting radiation modes, when calculated numerically. These radiation modes have been proven frequency-independent for two considered systems of fluidstructure interaction in 3 using an analytical formulation and are used in this study for experimental investigations.

This paper describes an experimental implementation of the global active control of sound radiation in an acoustic enclosure. The system of fluid-structure interaction consists of a rectangular plate coupled to a cuboid cavity. Section 2 describes the experimental setup. A set of harmonic velocities is measured by means of a scanning laser vibrometer (SLV) and included into an identified plant model. Radiation filters are then evaluated and appended to this plant. The system identification process as well as the control synthesis are described in detail in section 3 . An overview of the achieved global noise suppression as well as the control mechanisms is given in section 4. Section 5 concludes this study and gives an outlook to future research. 


\section{Experimental setup}

This section presents the experimental set-up of the plate-cavity system, as well as the measurement equipment and the active elements used. An exterior view of the experimental plate-cavity system is shown in Fig. 1a. The plate is made of $2 \mathrm{~mm}$ thick aluminium with the dimensions $\left(L_{x} \times L_{y}\right)=(0.6 \times 0.8) \mathrm{m}^{2}$. The plate edges are fixed in an aluminium frame of $15 \mathrm{~mm}$ thickness. A total of 24 accelerometers are distributed equally spaced across the plate. Additionally, a grid of $13 \times 15$ highly reflective dots are applied to the plate to facilitate the measurement of the surface velocity by a SLV.

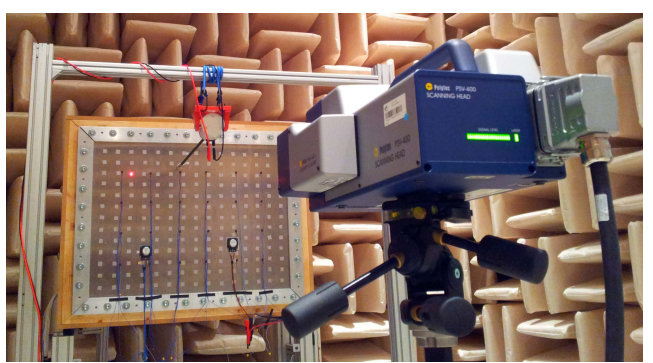

(a)

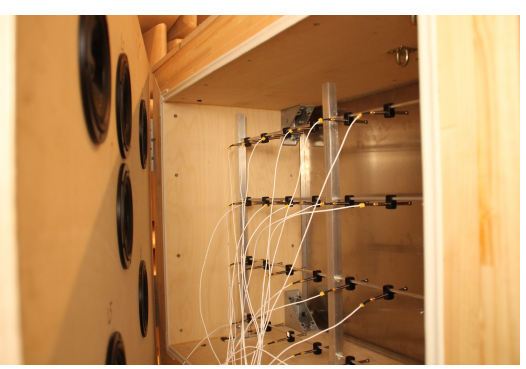

(b)

Figure 1: Experimental set-up of the plate-cavity system as seen from the outside (a) and the inside (b); the cavity includes loudspeakers and microphones for the evaluation of the acoustic potential energy

The hardware components for the active control system are listed in Table 1 The disturbance is induced by a primary shaker while two secondary shakers are used for the active suppression of sound radiation. During the experimental identification process, broadband uncorrelated signals for the primary and secondary shakers are generated by a DSPACE ${ }^{\circledR}$ rapid control prototyping system. The low-pass filtered accelerometer outputs are measured and the transfer functions estimated. The positions of the primary and secondary shakers as well as the sensors on the plate are summarized in Fig. 2. For the primary shaker the position is chosen not to coincide with a nodal line in the frequency range up to $500 \mathrm{~Hz}$, so that all modes of the plate can be excited. The positions of the secondary actuators were chosen taking into account controllability and observability criteria as stated by Gawronski [12].

Fig. 1 b shows an interior view of the acoustic cavity comprised of a wooden mock-up. The acoustic boundary is built as a double-plate system with additional damping in its enclosed cavity. The interior cavity of the experimental set-up has a depth of $L_{z}=0.42 \mathrm{~m}$. An array of $4 \times 8$ microphones is used for the acoustic measurements. The microphone array is shifted in six equally spaced positions along the cavity depth, so that a total of 192 pressure measurements is used for the calculation of the acoustic potential energy. It should be noted, that the microphones are not part of the control system, as they are solely used for the evaluation of the acoustic potential energy. The hardware components 
Table 1: Hardware components of the active control system

\begin{tabular}{lll}
\hline Device & Type & Additional data \\
\hline Accelerometer & PCB 352A24 & Mass $m=0.8 \mathrm{~g}$ \\
Primary Shaker & LDS V201 & - \\
Control Shaker & Visaton EX45 & Mass $m=60 \mathrm{~g}$ \\
$\begin{array}{l}\text { Real-Time } \\
\text { system }\end{array}$ & DSPACE ${ }^{\circledR}$ & Sampling frequency $F_{s}=1 \mathrm{kHz}$ \\
$\begin{array}{l}\text { Low-pass } \\
\text { filters }\end{array}$ & $\begin{array}{l}\text { Kemo Card } \\
\text { Master 255G }\end{array}$ & $\begin{array}{l}\text { Cut-off frequency } F_{c}=1 \mathrm{kHz}, \\
\text { Attenuation } 24 \mathrm{~dB} / \text { Octave }\end{array}$ \\
\hline
\end{tabular}

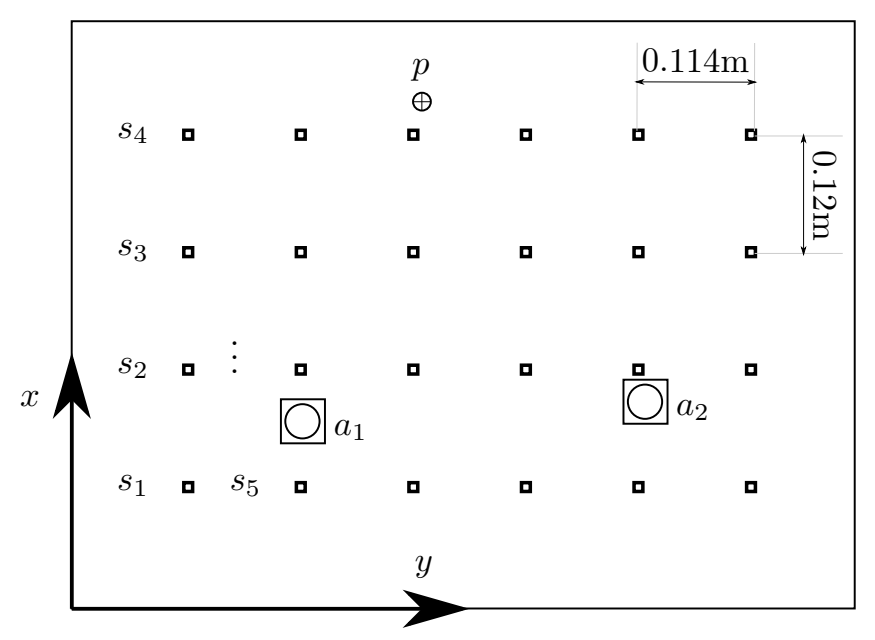

Figure 2: Positions of primary shaker $(p)$, actuators $\left(a_{m}\right)$ and sensors $\left(s_{n}\right)$

for the acoustic measurements are detailed in Table 2

\section{System identification and controller synthesis}

An overview of the methods used for the system identification and the controller design is given in this section. The identification of plant models, their extension with additional velocity outputs as well as radiation filter sensing are presented. Based on the extended plant model with the radiation filter output, the applied feedback control synthesis is described.

\subsection{Extended plant model}

The synthesis of feedback controllers necessitates accurate models of the plant to be controlled. For this study, a linear time-invariant discrete-time 
Table 2: Hardware components for structural and acoustic measurements

\begin{tabular}{ll}
\hline Device & Type \\
\hline $\begin{array}{l}\text { Scanning laser } \\
\text { doppler vibrometer }\end{array}$ & OFV-055 (scan unit), \\
OFV-3001-S (controller) \\
Microphones & PCB T130D21 \\
Data acquisition frontend & NI PXI-1042Q \\
\hline
\end{tabular}

state-space model $\mathbf{G}(z)$ is used, which is described at discrete time steps $n \in \mathbb{N}_{0}$ as

$$
\begin{gathered}
\mathbf{x}(n+1)=\mathbf{A} \mathbf{x}(n)+\mathbf{B u}(n) \\
\mathbf{y}(n)=\mathbf{C x}(n)+\mathbf{D u}(n)
\end{gathered}
$$

where $\mathbf{A}, \mathbf{B}, \mathbf{C}$ and $\mathbf{D}$ describe the system maxtrix, input matrix, output matrix and feedthrough matrix, respectively. Based on Eqs. 1a and (1b), the plant model $\mathbf{G}(z)$ can be defined as

$$
\mathbf{G}(z):=\left[\begin{array}{l|l}
\mathbf{A} & \mathbf{B} \\
\hline \mathbf{C} & \mathbf{D}
\end{array}\right]
$$

with the complex variable $z=\exp (i \omega T)$. Here, $\exp (\cdot)$ describes the exponential function, $i=\sqrt{-1}$ the complex unit, $\omega$ the angular frequency and $T$ the sampling period. For the control system shown in Fig. $1 \mathrm{a}, \mathbf{u} \in \mathbb{R}^{n_{u}}$ are the primary and secondary shaker inputs and $\mathbf{y} \in \mathbb{R}^{n_{y}}$ are the accelerometer outputs, with $n_{\{\cdot\}}$ describing the size of the vector $\{\cdot\}$. In Eqs. $1 \mathrm{a}$ and $\sqrt{1 \mathrm{~b}}, \mathbf{x} \in \mathbb{R}^{n_{x}}$ is the state vector. The matrices $\mathbf{A}, \mathbf{B}, \mathbf{C}$ and $\mathbf{D}$ of the state space model are identified using measured data of $\mathbf{u}$ and $\mathbf{y}$. For the system identification, the subspace identification method based on $\mathrm{QR}$ as well as singular value decomposition is used, the theory of which can be found e.g. in the textbook of Katayama [13. The singular values of the frequency response are shown in Fig. 3, comparing the identified plant using 150 states with the measured transfer functions.

Since a good agreement of the modelled and measured transfer paths is evident, this number of states is sufficient to model the plant dynamics accurately.

For the efficient active control of acoustic quantities, e.g. sound power or potential energy, over a wide frequency range a finer grid of measured data is needed than supplied by accelerometer measurements. In order to include additional velocity outputs in the control model, an extended plant [14, 15] is identified, based on SLV measurements. The identification process of the extended plant takes place as follows. Every shaker (primary and secondary) is excited individually as the point velocities are measured by the SLV sequentially. The resulting complex frequency response function (FRF) matrix $\mathbf{H}\left(z_{k}\right)$ links the three actuators to the 195 SLV measurement points. In order to include these points in the plant $\mathbf{G}$, additional state-space matrices $\mathbf{C}_{e x t}$ and $\mathbf{D}_{\text {ext }}$ are 


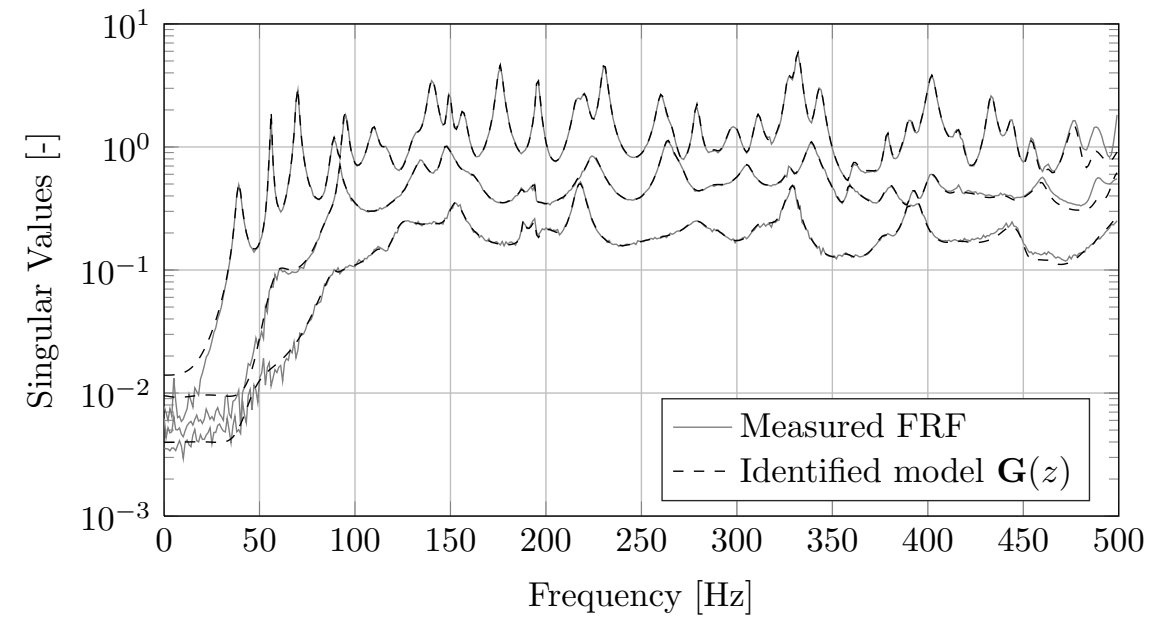

Figure 3: Comparison of singular values of the frequency response

calculated. Fixing the system matrix $\mathbf{A}$ and the input matrix $\mathbf{B}$ of the original plant model, yields the least-squares problem

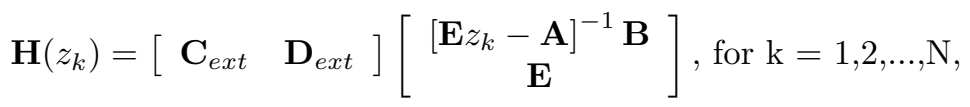

where $\mathbf{E}$ is the identity matrix. Eq. (3) needs to be solved for the unknown matrices $\mathbf{C}_{e x t}$ and $\mathbf{D}_{e x t}$ for all $N$ discrete frequency points. Appending the velocity outputs to the plant in Eqs. (1a) and (1b) results in the extended plant $\mathbf{G}_{\text {ext }}(\omega)$

$$
\begin{array}{r}
\mathbf{x}(n+1)=\mathbf{A} \mathbf{x}(n)+\mathbf{B u}(n) \\
{\left[\begin{array}{c}
\mathbf{y}_{\text {ext }}(n) \\
\mathbf{y}(n)
\end{array}\right]=\left[\begin{array}{c}
\mathbf{C}_{\text {ext }} \\
\mathbf{C}
\end{array}\right] \mathbf{x}(n)+\left[\begin{array}{c}
\mathbf{D}_{\text {ext }} \\
\mathbf{D}
\end{array}\right] \mathbf{u}(n),}
\end{array}
$$

with

$$
\mathbf{G}_{e x t}(\omega)=\left[\begin{array}{l|l}
\mathbf{A} & \mathbf{B} \\
\hline \mathbf{C}_{e x t} & \mathbf{D}_{e x t} \\
\mathbf{C} & \mathbf{D}
\end{array}\right] .
$$

The extended plant $\mathbf{G}_{\text {ext }}(\omega)$ allows the real-time estimation of the additional point velocities $\mathbf{y}_{\text {ext }}(n)$ by measuring the accelerometer outputs $\mathbf{y}$. In Fig. 4. the singular values of the additional part (excluding the sensor outputs) of the extended plant $\mathbf{G}_{\text {ext }}(\omega)$ are compared to the singular values of the FRF matrix $\mathbf{H}\left(z_{k}\right)$. A reasonable agreement is indicated.

\subsection{Interior radiation filters}

The interior radiation efficiencies and modes are calculated in [3], using an analytical formulation. For experimental application, the radiation modes of 


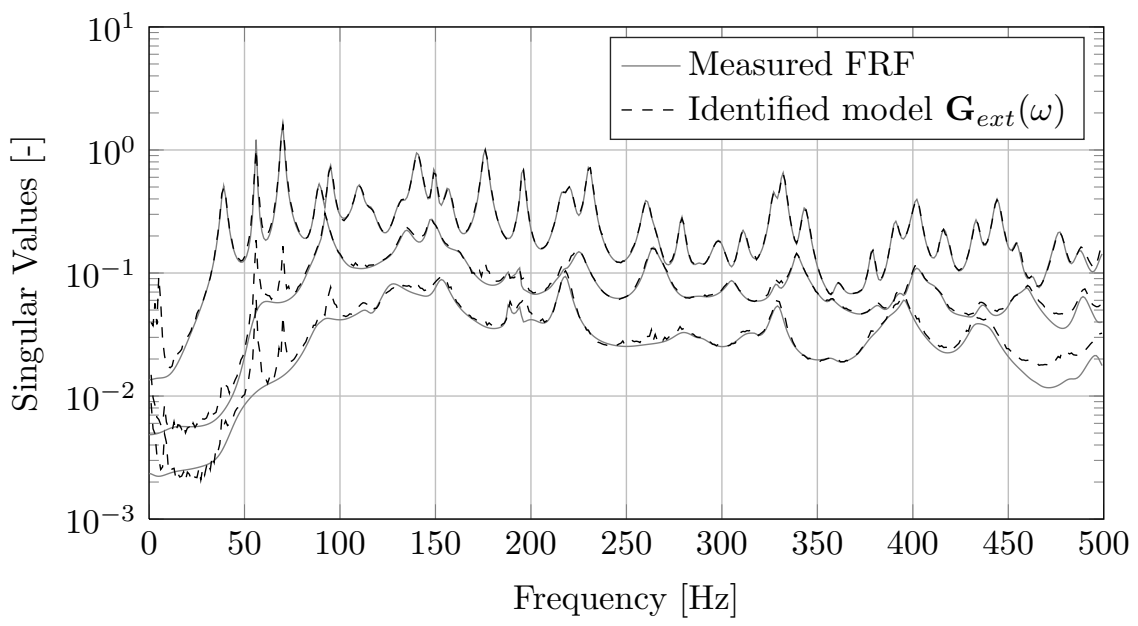

Figure 4: Comparison of singular values of the extended plant

the rectangular plate are evaluated at the SLV measurement points. Due to manufacturing tolerances of the experimental setup and temperature fluctuations, the eigenfrequencies and -forms as well as the damping characteristics of the acoustic medium will change compared to the analytical model. In this study, the eigenforms are assumed to be invariant.

It is shown in [3, that the acoustic potential energy $E(\omega)$ can be described in terms of the surrounding structural velocity $v(x, y, \omega)$ on the surface $S$. The acoustic potential energy $E(\omega)$ can be rewritten for the rectangular cavity as a function dependent on the interior radiation modes $u_{l, m}(x, y)$

$$
u_{l, m}(x, y)=\cos \left(\frac{l \pi x}{L_{x}}\right) \cos \left(\frac{m \pi y}{L_{y}}\right)
$$

and radiation efficiencies $s_{l, m}(\omega)$

$$
s_{l, m}(\omega)=\sum_{n=0}^{\infty} \frac{\rho c^{2}}{4 \Gamma_{l, m, n}}\left|\frac{i \omega}{\omega_{l, m, n}^{2}+2 i \zeta_{l, m, n} \omega_{l, m, n} \omega-\omega^{2}}\right|^{2}
$$

as

$$
E(\omega)=\sum_{l, m=1}^{\infty} s_{l, m}(\omega)\left|\int_{S} u_{l, m}(x, y) v(x, y, \omega) d S\right|^{2} .
$$

Here, $l, m$ and $n$ are the acoustic modal indices in the $x, y$ and $z$-direction respectively. The scalars $\rho$ and $c$ describe the fluid density and sound velocity. These are assumed as $\rho=1.204 \frac{\mathrm{kg}}{\mathrm{m}^{3}}$ and $c=343 \frac{\mathrm{m}}{\mathrm{s}}$. The volume normalization factors $\Gamma_{l, m, n}$ of the rectangular cavity modes are derived according to

$$
\Gamma_{l, m, n}=\frac{L_{x} L_{y} L_{z}}{8} \epsilon_{l} \epsilon_{m} \epsilon_{n}
$$


with

$$
\epsilon_{l}= \begin{cases}2 & , l=0 \\ 1 & , \text { otherwise }\end{cases}
$$

The angular eigenfrequencies $\omega_{l, m, n}$ and modal damping ratios $\zeta_{l, m, n}$ of the cavity in Eq. (7) are identified by means of a broadband loudspeaker excitation inside the cavity and measuring the resulting acoustic response using the microphones. For identification purposes, a loudspeaker excitation is preferred over a structural excitation, since using an acoustic source less structural vibration modes are excited and the cavity modes can be identified more distinctly. The resulting FRF matrix from the loudspeaker to the microphones is utilized, to identify the acoustic eigenfrequencies. Additionally, the corresponding modal damping ratios are calculated using the half-power bandwidth method [16. The values of the identified compared to the analytical eigenfrequencies according to 3 as well as the corresponding modal damping ratios are shown in Table 3 . It should be noted, that the $(0,0,0)$ cavity mode at $0 \mathrm{~Hz}$ can not be measured. However, the cavity mode can not be neglected, due to its high coupling to the $(1,1)$ structural mode. It has been observed, that the modal damping does not change the resulting radiation filter output to a high degree. The modal damping ratio of this cavity mode is therefore assumed as $1 \%$.

Using the identified eigenfrequencies and modal damping ratios, the radiation

Table 3: Identified acoustic eigenfrequencies

\begin{tabular}{llll}
\hline$(l, m, n)$ & Experimental Freq. & Analytical Freq. & Damp. rat. \\
\hline$(0,0,0)$ & $0 \mathrm{~Hz}$ & $0 \mathrm{~Hz}$ & $1.00 \%$ \\
$(0,1,0)$ & $211.9 \mathrm{~Hz}$ & $214.37 \mathrm{~Hz}$ & $2.65 \%$ \\
$(1,0,0)$ & $288.1 \mathrm{~Hz}$ & $285.83 \mathrm{~Hz}$ & $1.19 \%$ \\
$(1,1,0)$ & $369.4 \mathrm{~Hz}$ & $357.29 \mathrm{~Hz}$ & $1.60 \%$ \\
$(0,0,1)$ & $417.5 \mathrm{~Hz}$ & $408.33 \mathrm{~Hz}$ & $0.90 \%$ \\
$(0,2,0)$ & $429.4 \mathrm{~Hz}$ & $428.75 \mathrm{~Hz}$ & $0.80 \%$ \\
$(0,1,1)$ & $461.3 \mathrm{~Hz}$ & $461.18 \mathrm{~Hz}$ & $0.88 \%$ \\
$(1,0,1)$ & $497.5 \mathrm{~Hz}$ & $498.43 \mathrm{~Hz}$ & $0.69 \%$ \\
\hline
\end{tabular}

efficiencies are calculated according to Eq. (7). A total of five radiation modes dominates the considered frequency range. The radiation efficiencies are depicted in Fig. 5a.

The square root of each radiation efficiency $\sqrt{s_{l, m}(z)}$ is modelled as a minimum phase filter $S_{j}(z)$ by means of the Robust Control Toolbox [17 in Matlab ${ }^{\circledR}$. The singular values of the identified state-space model with the state-dimension four and the square root of the radiation efficiencies are shown in Fig. 5b. A good agreement of the identified model $S_{j}(z)$ with the square root of the radiation efficiencies is achieved. The radiation filter $\boldsymbol{r}_{j}(z)$ with index $j$ is calculated from a spatial discretisation of the radiation modes $\boldsymbol{u}_{j} \in \mathbb{R}^{195}$ as

$$
\boldsymbol{r}_{j}(z)=S_{j}(z) \boldsymbol{u}_{j}^{T}
$$




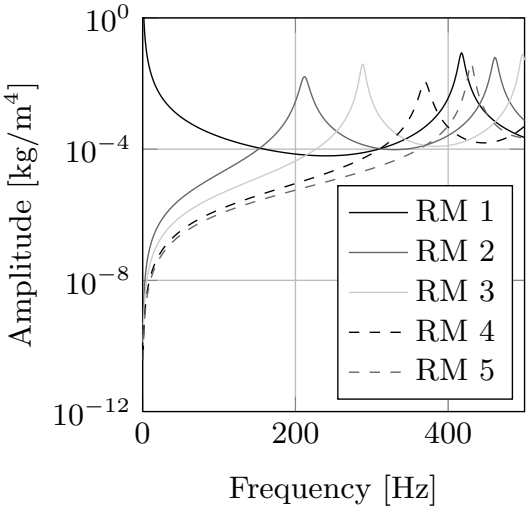

(a)

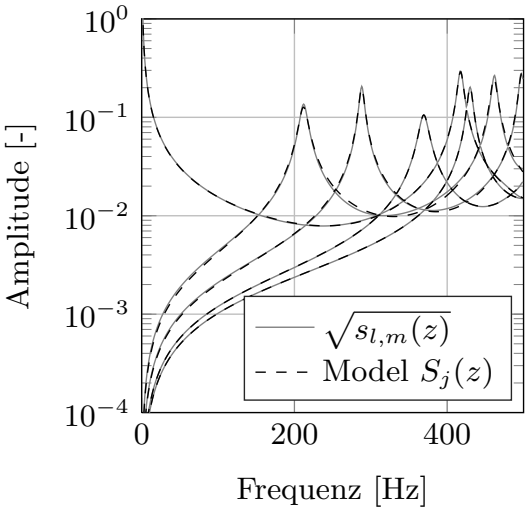

(b)

Figure 5: Radiation efficiencies (a) and comparison of singular values for the radiation filters (b)

Stacking of all radiation filters $\boldsymbol{r}_{j}(z)$ into a single system yields a rectangular transfer matrix $\mathbf{R}(z)$. The 195 velocity outputs $\mathbf{y}_{\text {ext }}(n)$ of the extended plant are multiplied by $\mathbf{R}(z)$, which leads to five outputs, the signal energy of which corresponds to the acoustic potential energy radiated by each radiation mode. With the five radiation filters, the acoustic potential energy in the enclosure is estimated based on the structural velocity. In order to get a reference for the estimation of the acoustic potential energy, a number of $t=192$ acoustic pressure values $\boldsymbol{p}$ inside the cavity are measured as described in section 2 . The acoustic potential energy $E_{r}(\omega)$ obtained by the measurements is calculated as

$$
E_{r}(\omega)=\frac{L_{x} L_{y} L_{z}}{4 \rho c^{2} t} \boldsymbol{p}^{H} \boldsymbol{p} .
$$

A comparison of the measurement $E_{r}(\omega)$ with the FRFs from the primary input to the acoustic potential energy for the case of radiation filter sensing at the SLV points is shown in Fig. 6. A qualitative agreement of the coupled system dynamics can be observed in the considered frequency range.

\section{3. $\mathcal{H}_{\infty}$ control}

This section describes the weighting scheme of the identified plant as well as the synthesis of the controller $\mathbf{K}$ used in this study. Fig. 7] shows the control concept, including the extended plant $\mathbf{G}_{\text {ext }}$ and the radiation filter $\mathbf{R}$ as mentioned in the preceding section. Subsequently, the frequency-dependence of the plant models is omitted for the sake of brevity. Due to the stochastic nature of the excitation, the feedback controller needs to provide broadband control performance. This is achieved by using a robust control approach [18.

The transfer functions of the plant $\mathbf{P}$ in Fig. 7 can be expressed as 


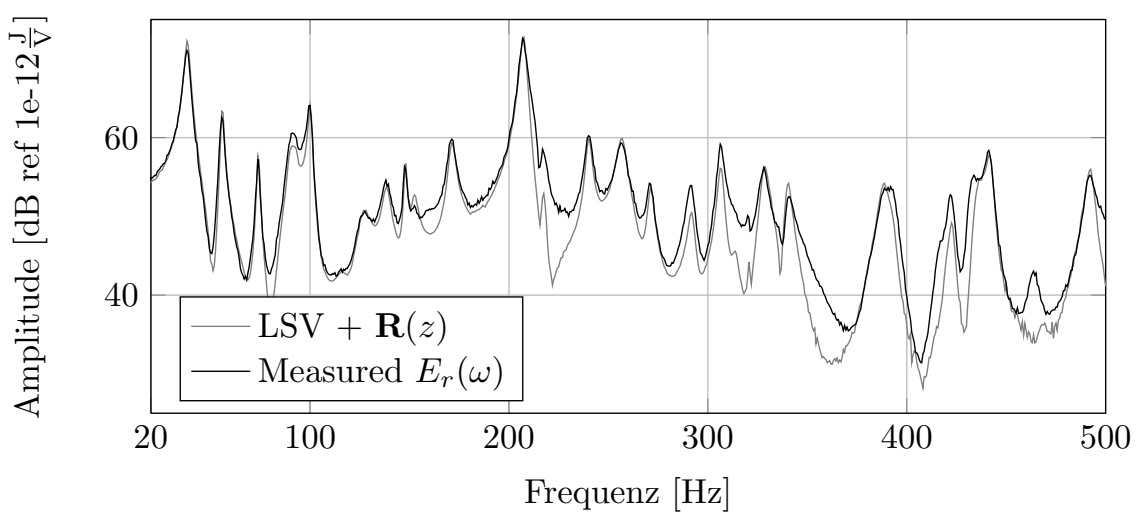

Figure 6: Comparison of FRFs from primary shaker to acoustic potential energy

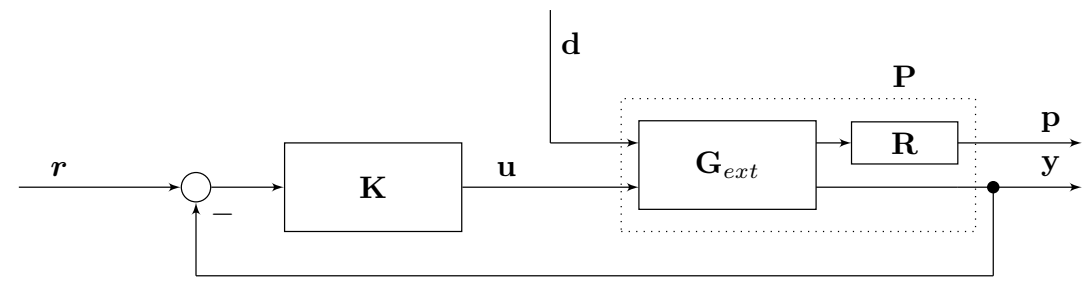

Figure 7: Control concept for feedback radiation filter control

$$
\left\{\begin{array}{l}
\mathbf{p} \\
\mathbf{y}
\end{array}\right\}=\mathbf{P}\left\{\begin{array}{l}
\mathbf{d} \\
\mathbf{u}
\end{array}\right\}=\left[\begin{array}{ll}
\mathbf{P}_{11} & \mathbf{P}_{12} \\
\mathbf{P}_{21} & \mathbf{P}_{22}
\end{array}\right]\left\{\begin{array}{l}
\mathbf{d} \\
\mathbf{u}
\end{array}\right\} .
$$

In Eq. (13), $\mathbf{d}$ describes the disturbance, $\mathbf{y}$ the sensor outputs, $\mathbf{u}$ the controller output and $\mathbf{p}$ the performance output.

The control objective for robust control is achieved by minimising the $\mathcal{H}_{\infty}$ norm of the transfer function from the disturbance $\mathbf{d}$ to the performance output $\mathbf{p}$ [18. By introducing a frequency-dependent weighting function $\mathbf{W}$, additional constraints for the quantities can be introduced. The weighting scheme for the closed control loop is shown in Fig. 8, consisting of four weighting filters. The calculation of the weighting filters is based on two variables, which are related to the rate of disturbance rejection and restriction of the control input [19. The aim of the $\mathcal{H}_{\infty}$ control synthesis is to find a controller $\mathbf{K}$ that limits the $\mathcal{H}_{\infty}$ norm of the plant $\mathbf{T}_{z w}$ below a value $\gamma$ :

$$
\left\|\mathbf{T}_{z w}\right\|_{\infty}=\sup _{\omega \in \mathbb{R}} \bar{\sigma}\left(\mathbf{T}_{z w}(i \omega)\right)<\gamma .
$$

Here, the operator sup describes the supremum over all real valued frequencies $\omega$ and $\bar{\sigma}$ is the maximum singular value. As can be seen from Fig. 8 , the transfer 


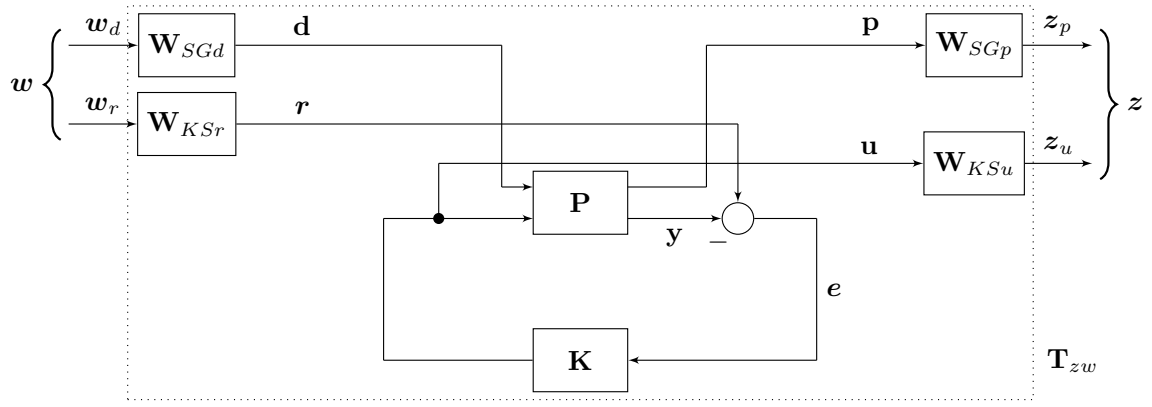

Figure 8: Weighting scheme for generalised plant

function $\mathbf{T}_{z w}$ is defined as

$$
\mathbf{T}_{z w}=\left[\begin{array}{cc}
\mathbf{W}_{S G p}\left(\mathbf{P}_{11}-\mathbf{P}_{12} \mathbf{K S P}_{21}\right) \mathbf{W}_{S G d} & \mathbf{W}_{S G p} \mathbf{P}_{12} \mathbf{K S W} \mathbf{W}_{K S r} \\
-\mathbf{W}_{K S u} \mathbf{K S P}_{21} \mathbf{W}_{S G d} & \mathbf{W}_{K S u} \mathbf{K S W} \mathbf{W}_{K S r}
\end{array}\right]
$$

with the sensitivity $\mathbf{S}$

$$
\mathbf{S}=\left[\mathbf{E}+\mathbf{P}_{22} \mathbf{K}\right]^{-1} .
$$

The term $\mathbf{P}_{11}-\mathbf{P}_{12} \mathbf{K} \mathbf{S P}_{21}$ describes the closed loop transfer path from the disturbance $\mathbf{d}$ to the performance output $\mathbf{p}$. The solution of the synthesis for the controller $\mathbf{K}$ is computed in Matlab ${ }^{\circledR}$ by means of the Robust Control Toolbox [17.

\section{Control results}

This section presents the control measurement results. The closed loop performance is evaluated in terms of the plate vibration and cavity noise reduction. The acoustic potential energy is estimated from the microphone measurement data according to Eq. (12). A comparison of the estimated acoustic potential energy for the open and closed loop measurements is shown in Fig. 9. Throughout the frequency bandwidth of $0-500 \mathrm{~Hz}$, a reduction of the sound radiation can be observed, with narrowband exceptions occurring at $270 \mathrm{~Hz}$ and $412 \mathrm{~Hz}$. Integrated over the frequency bandwidth, a reduction of $2.14 \mathrm{~dB}$ in acoustic potential energy is achieved. A maximum reduction of $14.31 \mathrm{~dB}$ is observed at $176.3 \mathrm{~Hz}$. The corresponding reduction in mean squared velocity level of the structural vibration is plotted in Fig. 10. Contrary to the acoustic potential energy, the mean velocity is not necessarily reduced in order to achieve a reduction in sound radiation. This phenomenon is also known from the active control of the far field radiated sound power 20 .

In order to evaluate the mechanisms involved in the reduction of sound radiation, Figs. 11 and 12 show exemplary operating deflection shapes of the structural velocity in the open and closed loop cases respectively. At $38.75 \mathrm{~Hz}$ it becomes evident, that mainly one structural mode contributes to the interior sound field. The resulting suppression of radiated noise is therefore attributable 


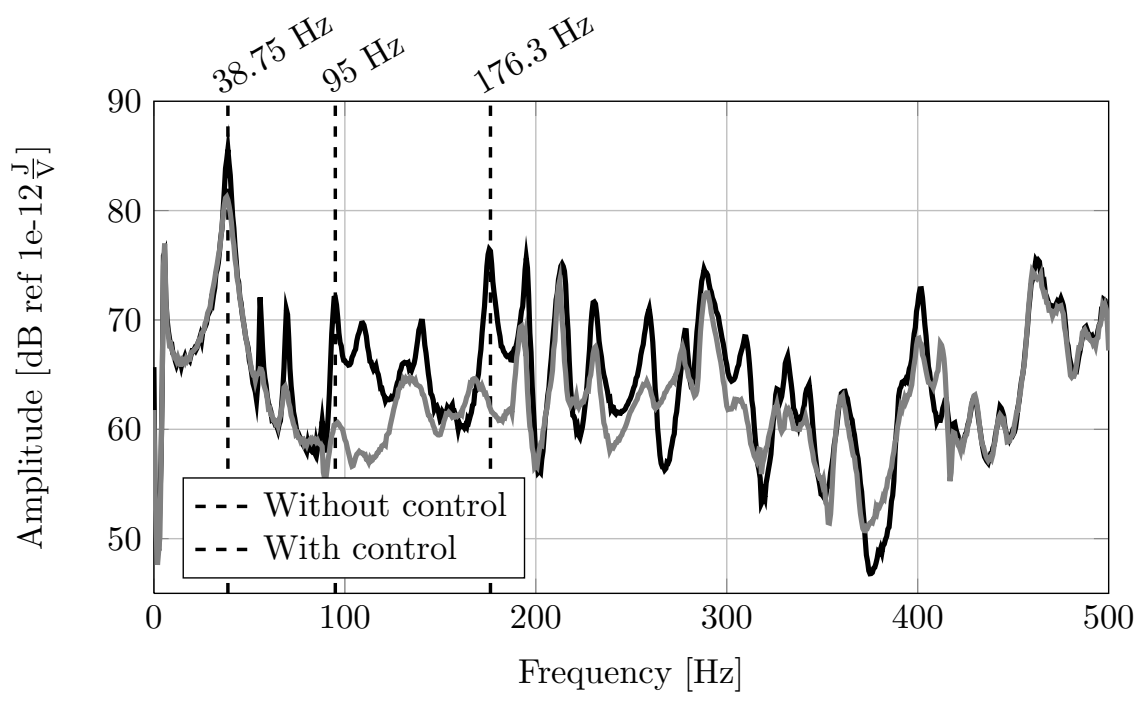

Figure 9: FRF from primary shaker to acoustic potential energy

to the reduction of this structural mode. This fact is emphasized by the equal reduction rate in acoustic potential energy (Fig. 9 ) and mean squared velocity (Fig. 10 of $\approx 5 \mathrm{~dB}$. At $95 \mathrm{~Hz}$ on the other hand, a reduction of interior noise radiation is achieved by a restructuring of the mode shape. The volume-displacing mode, similar to a $(1,3)$ mode, is converted to a non-volume-displacing $(2,2)$ mode. The class of non-volume-displacing modes are likely to be less efficiently coupled to interior enclosures [21]. Fig. 13 shows the corresponding magnitude sound pressure distribution inside the cavity at $95 \mathrm{~Hz}$, as measured by the microphone array according to section 2. A global reduction of the cavity noise is evident for the closed loop response in Fig. 13b,

The magnitude of the sound pressure distribution at $176.3 \mathrm{~Hz}$ for the open and closed loop is shown in Fig. 14, where a maximum reduction of the acoustic potential energy is achieved. At this frequency, the cavity response to the structural excitation is dominated by the acoustic $(0,1,0)$ mode with a nodal line at $0.4 \mathrm{~m}$. The closed loop response depicted in Fig. 14b shows a global reduction in sound pressure throughout the cavity.

\section{Conclusion}

The frequency-independent radiation modes of interior sound radiation are studied using an experimental coupled plate-fluid system. Radiation mode sensing is conducted through an extension of structural velocity measurements to the plant model. The estimated acoustic potential energy resulting from radiation mode sensing is compared to microphone measurements and a qualitative agreement is shown. The active control of the radiation filter outputs is then 


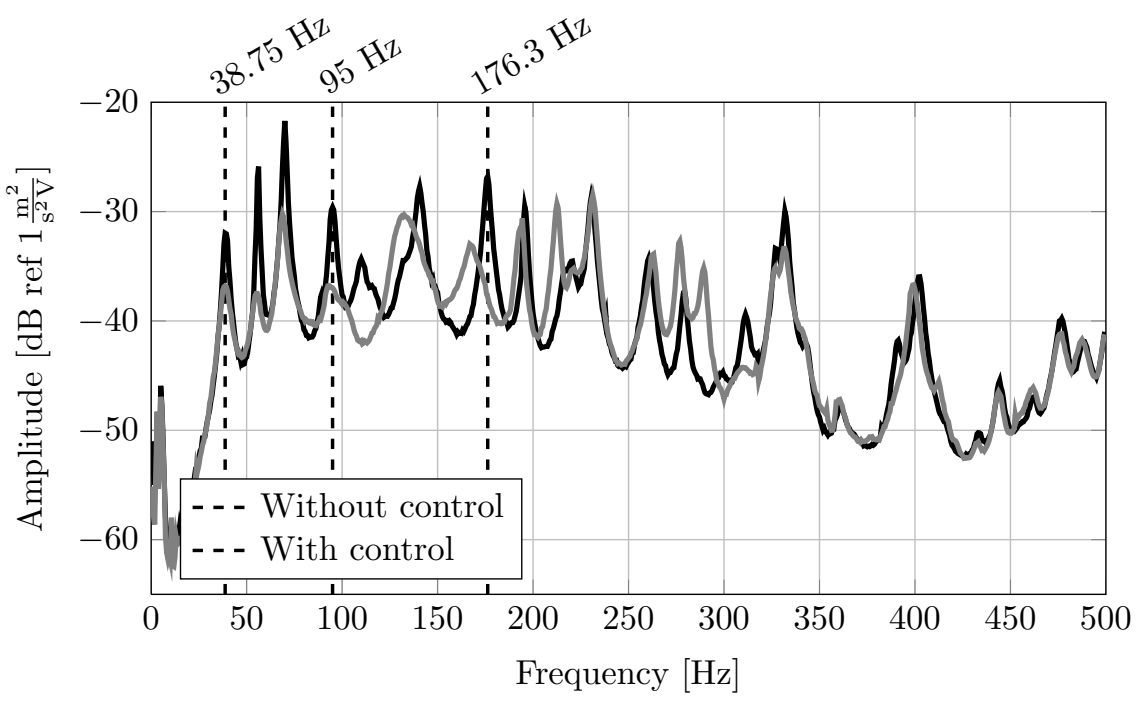

Figure 10: FRF from primary shaker to mean squared velocity

evaluated using a $\mathcal{H}_{\infty}$ controller. As a result, a broadband reduction of the acoustic potential energy is achieved under stochastic excitation. Based on structural and acoustic measurements, exemplary mechanisms involved in the global reduction of interior noise are presented.

Future work will be focussed on the active control of interior sound radiation including more realistic excitations as well as increasingly complex structurecavity systems. E.g. assuming an excitation of a turbulent boundary layer for aerospace applications, the disturbance path cannot be included into the plant model. Additionally, structural stiffeners as well as curvature effects need to be taken into consideration in the case of more complex structures.

\section{Acknowledgement}

This study has been conducted within the project Transferzentrum - MRO und Cabin Upgrade (project nr. LAHH 144), which is funded by the authority for Economy, Transport and Innovation of Freie und Hansestadt Hamburg.

\section{References}

[1] B. S. Cazzolato, C. H. Hansen, Active control of sound transmission using structural error sensing, J. Acoust. Soc. Am. 104 (1998) 2878-2889.

[2] B. S. Cazzolato, C. H. Hansen, Structural radiation mode sensing for active control of sound radiation into enclosed spaces, J. Acoust. Soc. Am. 106 (1999) 3732-3735. 


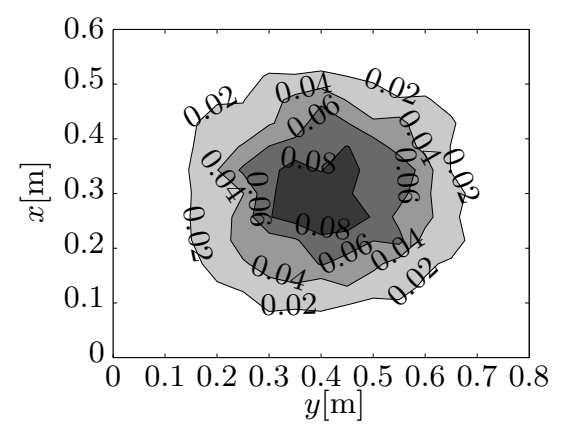

(a)

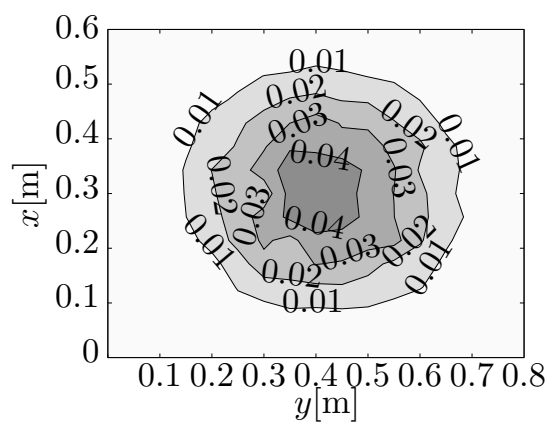

(b)

Figure 11: FRF from primary shaker to structural velocity (Unit: $[\mathrm{m} /(\mathrm{sV})]$ ) of the plate at $38.75 \mathrm{~Hz}$ without control (a) and with control (b)

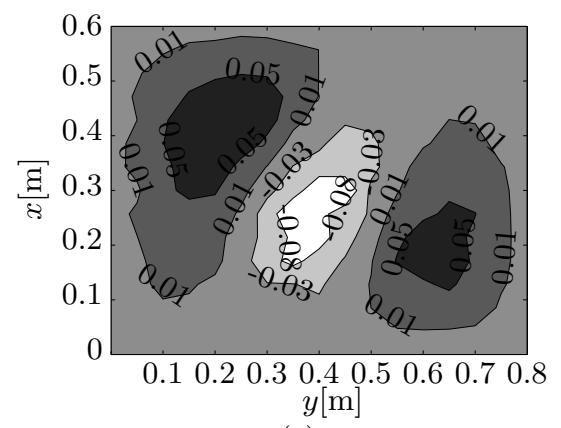

(a)

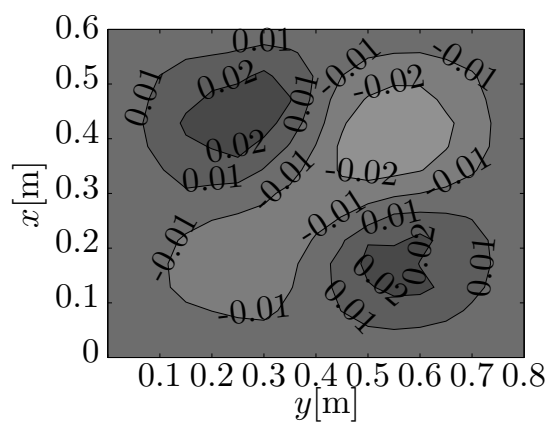

(b)

Figure 12: FRF from primary shaker to structural velocity (Unit: $[\mathrm{m} /(\mathrm{sV})]$ ) of the plate at $95 \mathrm{~Hz}$ without control (a) and with control (b)

[3] C. Hesse, J. V. Perez, M. Sinapius, Frequency-independent radiation modes of interior sound radiation: An analytical study, J. Sound Vib. 392 (2017) 31 - 40. doi:http://dx.doi.org/10.1016/j.jsv.2016.12.038. URL http://www.sciencedirect.com/science/article/pii/ S0022460X16307982

[4] S. D. Snyder, C. H. Hansen, The design of systems to control actively periodic sound transmission into enclosed spaces, part I: analytical models, J. Sound Vib. 170 (4) (1994) 433-449.

[5] S. D. Snyder, C. H. Hansen, The design of systems to control actively periodic sound transmission into enclosed spaces, part II: mechanisms and trends, J. Sound Vib. 170 (4) (1994) 451-472.

[6] J. Pan, C. H. Hansen, Active control of noise transmission through a panel 


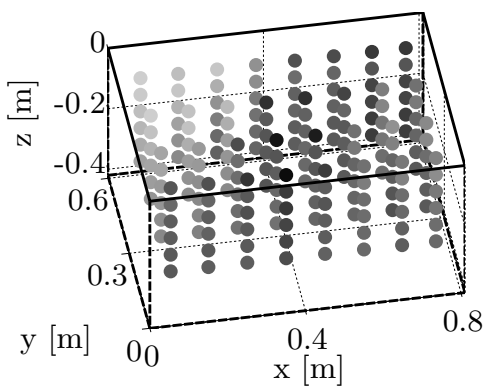

(a)

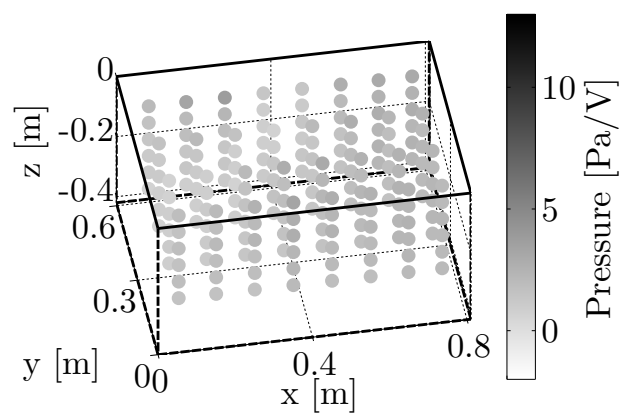

(b)

Figure 13: FRF from primary shaker to Sound pressure magnitude distribution at $95 \mathrm{~Hz}$ without control (a) and with control (b)

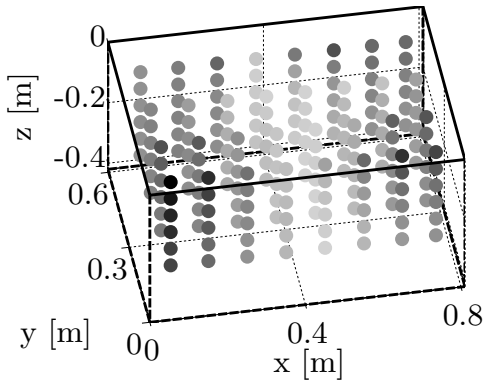

(a)

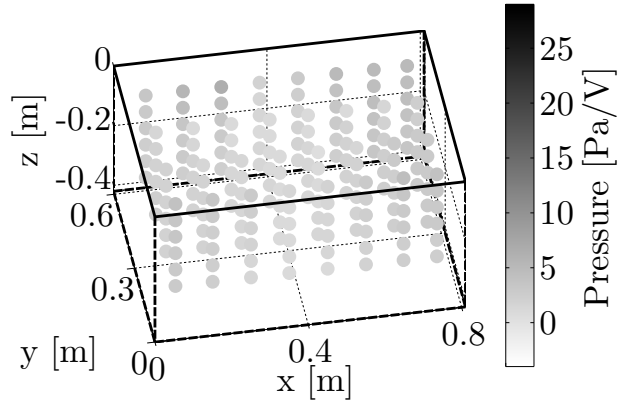

(b)

Figure 14: FRF from primary shaker to Sound pressure magnitude distribution at $176.3 \mathrm{~Hz}$ without control (a) and with control (b)

into a cavity. II: Experimental study, J. Acoust. Soc. Am. 90 (3) (1991) 1488-1492.

[7] L. P. de Oliveira, K. Janssens, P. Gajdatsy, H. Van der Auweraer, P. S. Varoto, P. Sas, W. Desmet, Active sound quality control of engine induced cavity noise, Mechanical systems and signal processing 23 (2) (2009) 476-488.

[8] S. Griffin, A. Weston, J. Anderson, Adaptive noise cancellation system for low frequency transmission of sound in open fan aircraft, Shock and Vibration 20 (5) (2013) 989-1000.

[9] B. S. Cazzolato, Sensing systems for active control of sound transmission into cavities, Ph.D. thesis, The University of Adelaide (1999).

[10] S. M. Kim, M. J. Brennan, A comparative study of feedforward control 
of harmonic and random sound transmission into an acoustic enclosure, J. Sound Vib. 226 (3) (1999) 549-571.

[11] S. M. Kim, M. J. Brennan, Active control of harmonic sound transmission into an acoustic enclosure using both structural and acoustic actuators, J. Acoust. Soc. Am. 107 (2000) 2523-2534.

[12] W. Gawronski, Actuator and sensor placement for structural testing and control, J. Sound Vib. 208 (1) (1997) 101-109.

[13] T. Katayama, Subspace methods for system identification, Springer Science \& Business Media, 2006.

[14] M. Misol, S. Algermissen, H. P. Monner, Experimental investigation of different active noise control concepts applied to a passenger car equipped with an active windshield, J. Sound Vib. 331 (10) (2012) 2209-2219.

[15] S. Algermissen, M. Misol, O. Unruh, O. Heintze, H. P. Monner, Robust control of turbulent boundary layer noise transmission through a stiffened panel, in: Proc. of International Congress on Sound and Vibration, Cairo, Egypt, 2010.

[16] H. Dresig, F. Holzweißig, L. Rockhausen, Maschinendynamik, Springer, 2005.

[17] G. Balas, R. Chiang, A. Packard, M. Safonov, Robust control toolbox. For Use with Matlab. User's Guide, Version 3 (2005).

[18] K. Zhou, J. C. Doyle, Essentials of robust control, Vol. 180, Prentice hall Upper Saddle River, NJ, 1998.

[19] S. Algermissen, M. Sinapius, Robust gain scheduling for smart-structures in parallel robots, in: Robotic Systems for Handling and Assembly, Springer, 2010, pp. 159-174.

[20] F. J. Fahy, P. Gardonio, Sound and structural vibration: radiation, transmission and response, Academic press, 2007.

[21] A. J. Pretlove, Free vibrations of a rectangular panel backed by a closed rectangular cavity by a closed rectangular cavity, J. Sound Vib. 2 (3) (1965) 197-209. 